\title{
Role of Admission test in predicting foetal outcome in low and high risk pregnancies
}

\author{
Shailesh B. Patil ${ }^{1}$, Milind B Patil ${ }^{2 * *}$ \\ ${ }^{1}$ Associate Professor, ${ }^{2}$ Assistant Professor, Dept. of Obstetrics and Gynaecology, Ashwini Rural Medical College, Hospital \& \\ Research Centre, Kumbhari, Solapur, Maharashtra, India
}

*Corresponding Author:

Email: drmilindpatil@gmail.com

\begin{abstract}
Introduction: Unfortunately, risk assessment profiles used at present are insufficient. Intrapartum mortality and morbidity are not uncommon in the low risk pregnancies also. FHR and fetal acidosis can occur with the same frequency as in a High risk group. Hence, there is a need for a simple, effective screening test to identify the pregnancies requiring continuous EFM.

Materials and Methods: This study is a prospective study comprising of 200 singleton pregnancies with cephalic presentation in early labour admitted to the labour room. Following Admission, the Admission test was done and the results were classified based on the three tier Heart interpretation system recommended by the 2008 NICHD Workshop on EFM as Reassuring, Nonreassuring and Ominous. The patients were then followed up and the mode of delivery and the different variables of perinatal outcome noted and correlated with the Admission test results.

Results: The Low risk group had 88\% Reassuring, 8\% Nonreassuring and 4\% Ominous Admission Test patterns. The High risk group had $80 \%$ Reassuring, 11\% Nonreassuring and 9\% Ominous Admission Test patterns. The study showed that operative deliveries were more significantly associated with Non reassuring and Ominous Admission test patterns especially in the High risk group. Admission test showed Sensitivity of $60 \%$, Specificity of $90.86 \%$, PPV $48.39 \%$ and NPV of $94.08 \%$.

Conclusion: Admission test can be used as an important non-invasive method to diagnose fetal compromise present at the time of admission in both high as well as low risk patients in labour.
\end{abstract}

Keywords: Admission test, Low and high risk pregnancies, Fetal distress, NICU admission.

\section{Introduction}

Parturition has been believed to be perhaps the most stressful situation for a human being to endure, much more than any other situation in his entire extra uterine life. ${ }^{1}$ It has been observed that intra partum fetal hypoxia is one of the potential factors involved in the development of handicaps and perinatal deaths. The goal is to identify the foetus at risk by antepartum and intrapartum foetal monitoring. ${ }^{2}$

Routine electronic monitoring of fetal heart rate in labour has become an established obstetric practice in the western world. ${ }^{3}$ However in developing countries like India, economic constraints limit routine monitoring. In labour wards with few monitors, selection of patients for continuous electronic fetal monitoring (EFM) and intermittent auscultation is necessary. At present, antenatal risk classification is used for this purpose, which recommends high risk patients for continuous EFM. ${ }^{3}$ Unfortunately risk assessment profiles are often an insufficient tool for selection. Intrapartum fetal morbidity is not uncommon in a low risk population. Fetal heart changes and fetal acidosis might occur with same frequency in a low risk group as in high risk group. ${ }^{3}$ Admission CTG has some value and more number of RCTs and other observational studies are required to hold up this view in case of high risk pregnancy. Do not recommend admission CTG in low-risk women as British guidelines published in 2001, whereas Swedish guidelines in 2001 recommend the test in all women. ${ }^{4,5}$ The method was evaluated and came to conclusion that, for assessing the current status of the fetus, the method was useful. Further, it was made remarks that this test could be a predictor of the neonate's wellbeing. ${ }^{6}$ Whereas in some studies shows that, in improving neonatal outcomes, this test is inefficient. ${ }^{7-9}$

The labour admission test is a screening test used in early labour to detect compromised fetuses on admission and to select the women in need of continuous Electronic Fetal Monitoring during labour. ${ }^{10}$

Admission Test is a short recording of FHR done on admission in labour, for a period of $15-20$ minutes by external Electronic Fetal Monitoring. It records graphically the fetal heart rate activity and uterine contractions in the same time, simultaneously and continuously, through uterine quiescence and contractions with fetal movements. Admission Test is not only simple, inexpensive, it is also non-invasive, can be easily performed and interpreted. It also consumes less time and has no contraindications for testing and more importantly can be used to screen a large number of populations quickly in an outpatient basis, even by a trained paramedical staff.

Our study is intended to evaluate the efficacy of the Admission Test in predicting the compromised fetuses, in both low risk and high risk patients.

\section{Objectives}

To evaluate the efficacy of Admission test in predicting fetal outcome. 


\section{Materials and Methods}

This prospective study comprises of 200 patients (100 low risk and 100 high risk cases) with singleton pregnancies with cephalic presentation in early labour, admitted to the labour room and fulfilling selection criteria were taken for study.

\section{Inclusion criteria:}

1. More 37 weeks of gestation.

2. Patients in first stage of labour.

3. Singleton pregnancies.

4. Both low risk and high risk pregnancies.

\section{Exclusion criteria:}

1. Multiple gestation.

2. Malpresentations. (Tranverse lie, Face presentation)

3. Congenital anamolies.

4. Patients with spurious labour, delivering > 24 hours after admission.

5. Patients in second stage of labour.

High risk group included patients with, preeclampsia, anaemia, gestational diabetes mellitus, cardiac disease, thyroid disease, renal disease, bad obstetric history, post-dated pregnancies, oligohydramnios, polyhydramnios, IUGR, $\mathrm{Rh}^{\text {-ve }}$ pregnancies, PROM, grand multipara, elderly primigravida.

Following admission, the patient details such as age, occupation, booked / unbooked case were noted down. Accurate gestational age was determined from a detailed menstrual history and USG estimation of gestational age. Detailed Obstetric history taken, any risk factors if present were identified. General physical examination, systemic examination, perabdominal and pervaginal examination done. Investigation reports and USG reports analyzed and risk factors if present were noted down. The patient was counseled about the Admission test and a written informed consent taken. The patient was made to lie down in the bed with $15^{\circ}$ left lateral tilt in order to avoid aorto - caval compression. This was achieved by placing a pillow underneath the right hip. The exact site where Fetal Heart Sound (FHS) was distinctly heard was localised with stethoscope and the USG (FHR) Transducer was placed at that site, after applying an aquasonic jelly and fastened with belt. The Toco transducer was also belted on the top of the abdomen over the uterine fundus to detect uterine contractions if any. The patient was made to hold the event marker and asked to press the button with each fetal movement. The reading was taken for a period of 20 minutes.

The Fetal Heart traces obtained were categorized as REASSURING (NORMAL), NONREASSURING (INDETERMINATE) AND OMINOUS according to the recommendations of Three tier Heart system recommended by the 2008 NICHD Workshop on EFM. Cases with Reassuring / Normal Admission test were followed by intermittent auscultation.

Cases with Ominous Admission test, intervention was done by:

1. Operative vaginal delivery (Forceps/ Ventouse)

2. Ceasserian section.

Cases with Non-reassuring / Indeterminate Admission test, a 20 min extension strip was obtained after the following:

1. Repositioning of patient in left lateral position.

2. Discontinuation of uterine stimulants.

3. Vaginal examination.

4. Administering of oxygen.

5. Correction of maternal dehydration.

If the Admission test reverted back to normal pattern, then labour was allowed to progress with continuous EFM and if it progressed to ominous pattern, then labour was terminated by intervention. At the time of delivery, data variables such as fetal distress during labour, ceasserian section for fetal distress, five minute apgar score less than 7, meconium stained liquor need for NICU admission were collected.

The mother and the baby were followed up until discharge from the hospital.

\section{Statistical Analysis}

Descriptive and inferential statistical analysis has been carried out in the present study. Results on continuous measurements are presented on Mean \pm SD and results on categorical measurements are presented in Number (\%). Significance is assessed at $5 \%$ level of significance. Chi-square/ Fisher Exact test has been used to find the significance of study parameters on categorical scale between two or more groups. Diagnostic statistics viz. Sensitivity, Specificity, PPV, NPV and Accuracy have been computed to find the correlation of Admission test to predict the perinatal outcome.

\section{Results}

Table 1: Age distribution

\begin{tabular}{|l|c|c|c|c|}
\hline \multirow{2}{*}{ Age in years } & \multicolumn{2}{|c|}{ Low risk } & \multicolumn{2}{c|}{ High risk } \\
\cline { 2 - 5 } & No & \% & No & \% \\
\hline $18-20$ & 22 & 22.0 & 18 & 18.0 \\
\hline $21-25$ & 40 & 40.0 & 37 & 37.0 \\
\hline $26-30$ & 30 & 30.0 & 34 & 34.0 \\
\hline $31-35$ & 06 & 6.0 & 10 & 10.0 \\
\hline
\end{tabular}




\begin{tabular}{|l|c|c|c|c|}
\hline$\geq 36$ & 02 & 2.0 & 01 & 1.0 \\
\hline Total & 100 & 100.0 & 100 & 100.0 \\
\hline Mean \pm SD & \multicolumn{2}{|c|}{$24.03 \pm 4.15$} & \multicolumn{2}{|c|}{$25.06 \pm 4.19$} \\
\hline
\end{tabular}

The above table shows maximum number of patients lie in the age group of 21-25 yrs, constituting $40 \%$ and $37 \%$ in low risk and high risk group respectively. Mean \pm SD in Low risk group is $24.03 \pm 4.15$. Mean \pm SD in High risk group is $25.06 \pm 4.19$.

Table 2: Distribution of Admission Test results

\begin{tabular}{|l|c|c|c|c|}
\hline \multirow{2}{*}{ AT results } & \multicolumn{2}{|c|}{ Low risk } & \multicolumn{2}{c|}{ High risk } \\
\cline { 2 - 5 } & No & \% & No & \% \\
\hline Reassuring & 88 & 88.0 & 80 & 80.0 \\
\hline Nonreassuring & 08 & 8.0 & 11 & 11.0 \\
\hline Ominous & 04 & 4.0 & 09 & 9.0 \\
\hline Total & 100 & 100.0 & 100 & 100.0 \\
\hline
\end{tabular}

In Low risk group; out of 100 cases, $88 \%$ had Reassuring, $8 \%$ had Non reassuring, $4 \%$ had Ominous Admission test patterns. In High risk group; out of 100 cases, $80 \%$ had Reassuring, $11 \%$ had Non reassuring and $9 \%$ had Ominous Admission test patterns.

Table 3: Correlation of AT Results with incidence of Meconium stained liquor, Apgar $<7$ @ 5 mins, and NICU admissions in Low and High risk pregnancies

\begin{tabular}{|c|c|c|c|c|c|c|c|}
\hline \multirow[t]{2}{*}{ AT results } & \multirow{2}{*}{$\begin{array}{c}\text { Number } \\
\text { of } \\
\text { patients }\end{array}$} & \multicolumn{2}{|c|}{ MSL } & \multicolumn{2}{|c|}{ Apgar $<7 @ 5$ mins } & \multicolumn{2}{|c|}{ NICU admissions } \\
\hline & & $\begin{array}{c}\text { Number } \\
\text { of } \\
\text { patients }\end{array}$ & Incidence & $\begin{array}{c}\text { Number } \\
\text { of } \\
\text { patients }\end{array}$ & Incidence & $\begin{array}{c}\text { Number } \\
\text { of } \\
\text { patients }\end{array}$ & Incidence \\
\hline Low Risk patients & 100 & 9 & 9.0 & 3 & 3.0 & 5 & 5.0 \\
\hline 1. Reassuring & 88 & 4 & 5.0 & 1 & 1.1 & 3 & 3.4 \\
\hline 2. Non Reassuring & 8 & 3 & 27.7 & 1 & 12.5 & 1 & 12.5 \\
\hline 3. Ominous & 4 & 2 & 50.0 & 1 & 25.0 & 1 & 25.0 \\
\hline High Risk Patients & 100 & 13 & 13.0 & 3 & 3.0 & 6 & 6.0 \\
\hline 4. Reassuring & 80 & 4 & 5.0 & 1 & 1.25 & 3 & 3.8 \\
\hline 5. $\quad$ Non Reassuring & 11 & 4 & 36.4 & 1 & 9.1 & 2 & 18.2 \\
\hline 6. Ominous & 09 & 5 & 55.6 & 1 & 11.1 & 1 & 11.1 \\
\hline
\end{tabular}

In 100 Low risk cases; Meconium stained liquor was found in 4 out of 88 (5\%) Reassuring admission tests, 3 out of $8(27.7 \%)$ Non reassuring admission tests and 2 out of $4(50 \%)$ Ominous Admission tests.

In 100 High risk cases; Meconium stained liquor was found in 4 out of $80(5 \%)$ Reassuring admission tests, 4 out of $11(36.4 \%)$ Non reassuring admission tests and 5 out of 9 (55.6\%) Ominous admission tests.

In 100 Low risk cases, Apgar score $<7$ @ 5 mins was found in 1 out of 88 (1.1\%) Reassuring admission tests, 1 out of $8(12.5 \%)$ Non reassuring admission tests and 1 out of 4 (25\%) Ominous admission tests.

In 100 High risk cases, Apgar score $<7$ @ 5 mins was found in 1 out of $80(1.25 \%)$ Reassuring admission tests, 1 out of $11(9.1 \%)$ Non reassuring admission tests and 1 out of $9(11.1 \%)$ Ominous admission tests.

In 100 Low risk cases: Out of 88 Reassuring admission tests, 3 (3.4\%) babies were admitted to NICU for observation, discharged within 24 hours. Out of 8 non reassuring admission tests, $1(12.5 \%)$ was admitted to NICU for meconium aspiration syndrome, discharged after 3 days. Out of 4 Ominous admission tests, 1 (25\%) baby was admitted to NICU for meconium aspiration, discharged 5 days later from NICU.

In 100 High risk cases: Out of 80 Reassuring admission tests, 3(3.8\%) babies were admitted to NICU for observation, discharged within $24-48$ hours. Out of 11 Non reassuring admission tests, $2(18.2 \%)$ babies were admitted to NICU for meconium aspiration syndrome, discharged after 3 days. Out of 9 Ominous admission tests, 1 $(11.1 \%)$ baby was admitted to NICU for meconium aspiration, discharged 5 days later from NICU.

Table 4: Diagnostic Performance of Admission test in predicting FD, MSL, Apgar score and NICU admissions

\begin{tabular}{|l|c|c|c|c|c|c|}
\hline & Sensitivity & Specificity & PPV & NPV & Accuracy & P value \\
\hline Low Risk patients & & & & & & \\
\hline AT to predict FD & 62.50 & 92.39 & 41.67 & 96.59 & 90.00 & $<0.001^{* *}$ \\
\hline
\end{tabular}




\begin{tabular}{|l|c|c|c|c|c|c|}
\hline AT to predict MSL & 55.56 & 92.31 & 41.67 & 95.46 & 89.00 & $<0.001^{* *}$ \\
\hline AT to predict Apgar $<7$ & 66.67 & 89.69 & 16.67 & 90.86 & 89.00 & $0.003^{* *}$ \\
\hline AT to predict NICU admn & 40.00 & 84.16 & 11.11 & 96.59 & 82.00 & 0.160 \\
\hline High Risk Patients & & & & & & \\
\hline AT to predict FD & 58.82 & 89.16 & 52.63 & 91.36 & 84.00 & $<0.001^{* *}$ \\
\hline AT to predict MSL & 69.23 & 87.36 & 45.00 & 95.00 & 85.00 & $<0.001^{* *}$ \\
\hline AT to predict Apgar $<7$ & 66.67 & 81.44 & 10.00 & 98.75 & 81.80 & $0.040^{*}$ \\
\hline AT to predict NICU admn & 50.00 & 81.91 & 15.00 & 96.25 & 80.00 & $0.050^{*}$ \\
\hline
\end{tabular}

In Low risk group: Correlation of Admission test in prediction of fetal distress shows Sensitivity - 62.50\%, Specificity $-92.39 \%$, PPV- 41.67\%, NPV- 96.59\%, Accuracy - 90\%.

Correlation of Admission test in prediction of MSL shows Sensitivity $-55.56 \%$, Specificity $-92.31 \%$, PPV41.67\%, NPV- 95.46\%, Accuracy - 89\%.

Correlation of Admission test in prediction of Apgar $<7$ @ mins shows Sensitivity - 66.67\%, Specificity 89.69\%, PPV- $16.67 \%$, NPV- 90.86\%, Accuracy - 89\%.

Correlation of Admission test in prediction of NICU admission shows Sensitivity $-40 \%$, Specificity $-84.16 \%$, PPV- $11.11 \%$, NPV- 96.59\%, Accuracy - 82\%.

In the High risk group: Correlation of Admission test in prediction of Fetal distress shows Sensitivity - 58.82\%, Specificity $-89.16 \%$, PPV- $52.63 \%$, NPV- $91.36 \%$, Accuracy $-84 \%$.

Correlation of Admission test in prediction of MSL shows Sensitivity $-69.23 \%$, Specificity $-87.36 \%$, PPV- $45 \%$, NPV- 95\%, Accuracy - 85\%.

Correlation of Admission test in prediction of Apgar $<7 @ 5$ mins shows Sensitivity $-66.67 \%$, Specificity 81.44\%, PPV- 10\%, NPV- $98.75 \%$, Accuracy - $81.80 \%$.

Correlation of Admission test in prediction of NICU admission shows Sensitivity - 50\%, Specificity $-81.91 \%$, PPV- $15 \%$, NPV- $96.25 \%$, Accuracy $-80 \%$.

Table 5: Comparison of Efficacy of Admission test in predicting the MSL, Apgar score $<7$ @ mins, NICU admissions (Low and High risk patients)

\begin{tabular}{|l|c|c|c|c|c|l|}
\hline \multirow{2}{*}{ Admission test } & \multicolumn{2}{|c|}{ Reactive } & \multicolumn{2}{c|}{ Non-reactive } & \multirow{2}{*}{ p-value } & \multirow{2}{*}{ Remarks } \\
\cline { 2 - 5 } & No & $\mathbf{\%}$ & No & \% & & \\
\hline MSL $(\mathrm{n}=22)$ & 8 & 36.4 & 14 & 63.6 & \multirow{2}{*}{$\mathrm{P}<0.001$} & \multirow{2}{*}{ Significant } \\
\hline NO MSL $(\mathrm{n}=178)$ & 160 & 89.9 & 18 & 10.1 & & \\
\hline Apgar $<7(\mathrm{n}=6)$ & 2 & 33.3 & 4 & 66.7 & $\mathrm{P}=0.001$ & \multirow{2}{*}{ Significant } \\
\hline Apgar $>7(\mathrm{n}=194)$ & 166 & 85.6 & 28 & 14.4 & & \\
\hline NICU $(\mathrm{n}=11)$ & 6 & 54.5 & 5 & 45.5 & $\mathrm{P}=0.006$ & \multirow{2}{*}{ Significant } \\
\hline Non NICU $(\mathrm{n}=189)$ & 162 & 85.7 & 27 & 14.3 & & \\
\hline
\end{tabular}

Out of 200 cases, consisting of both low and high risk pregnancies, 22 cases had meconium stained liquor. Out of 22 cases, $14(63.6 \%)$ had abnormal (i.e NRA+ Ominous) Admission test results.

The study shows that Abnormal AT is significantly associated with MSL with P value $<0.001 * *$

Out of 200 cases, consisting of both low and high risk pregnancies, 6 cases had Apgar score $<7$ @ mins. Out of 6 cases, 4 (66.7\%) had abnormal (i.e NRA+ Ominous) Admission test results.

The study shows that Abnormal AT is significantly associated with Apgar score $<7$ @ 5 mins with P value $<0.001 * *$.

Out of 200 cases, consisting of both low and high risk pregnancies, babies of 11 cases had NICU Admissions. Out of 11 cases, 5 (45.5\%) had abnormal (i.e NRA+ Ominous) Admission test results.

The study shows that Abnormal AT is significantly associated with NICU Admissions with P value $<0.001^{* *}$.

Table 6: Diagnostic Performance of Admission test in predicting FD, MSL, Apgar score, NICU admissions (Overall)

\begin{tabular}{|l|c|c|c|c|c|c|}
\hline & Sensitivity & Specificity & PPV & NPV & Accuracy & P value \\
\hline AT to predict FD & 60.00 & 90.86 & 48.39 & 94.08 & 87.00 & $<0.001^{* *}$ \\
\hline AT to predict MSL & 63.64 & 89.89 & 43.75 & 95.24 & 87.00 & $<0.001^{* *}$ \\
\hline AT to predict Apgar $<7$ & 66.67 & 85.57 & 12.50 & 98.01 & 85.00 & $0.005^{* *}$ \\
\hline AT to predict NICU & 45.45 & 85.71 & 15.63 & 96.43 & 83.50 & $0.006^{* *}$ \\
\hline
\end{tabular}


The Correlation of Admission test in prediction of fetal distress shows Sensitivity $-60 \%$, Specificity $-90.86 \%$, PPV- 48.39\%, NPV- 94.08\%, Accuracy - 87\%.

The Correlation of Admission test in prediction of MSL shows Sensitivity $-63.64 \%$, Specificity $-89.89 \%$, PPV- 43.75\%, NPV- 95.24\%, Accuracy - 87\%.

The Correlation of Admission test in prediction of Apgar $<7$ @ 5 mins shows Sensitivity $-66.67 \%$, Specificity - $85.57 \%$, PPV- $12.5 \%$, NPV- $98.01 \%$, Accuracy - 85\%.

The Correlation of Admission test in prediction of NICU admission shows Sensitivity $-45.45 \%$, Specificity 85.71\%, PPV- $15.63 \%$, NPV- $96.43 \%$, Accuracy $-83.50 \%$.

\section{Discussion}

The present study is a prospective study in which 100 low risk and 100 high risk pregnant women in early labour were randomly selected, the Admission test was performed and the efficacy of Admission test in the detection of perinatal outcome was studied.

In Aparna Hedge et al study, out of 200 cases, 84.5\% had Reassuring and $15.5 \%$ had Abnormal (Non reassuring + Ominous) Admission test patterns. ${ }^{1}$

In Buckshee et al study, out of 100 cases, $85 \%$ had Reassuring and $15 \%$ had Abnormal (Non reassuring + Ominous) Admission test patterns. ${ }^{11}$

In Vinita das et al study, out of 175 cases, $73.7 \%$ had Reassuring and 26.3\% had Abnormal (Non reassuring + Ominous) Admission test patterns. ${ }^{12}$

The present study, out of 200 cases, $84 \%$ had Reassuring and $16 \%$ had Abnormal (Non reassuring + Ominous) Admission test patterns.

The Present study results are correlating well with the results of Aparna Hegde et $\mathrm{al}^{1}$ and Buckshee et al studies. ${ }^{11}$

In the Geetha et al study, 100 cases (including both high and low risk) were studied. In the Reassuring group, 8 out of 73 i.e $11 \%$ underwent LSCS, of which 2 (25\%) had LSCS done for fetal distress. In patients with abnormal Admission test results, 18 out of 27 i.e $66.7 \%$ had caesarian deliveries, out of which $15(83.33 \%)$ had LSCS done for fetal distress. ${ }^{13}$

In the present study, in the Reassuring group , 29 out of 168 i.e $17.26 \%$ underwent LSCS, of which 8 (27.58\%) had LSCS done for fetal distress. In patients with abnormal Admission test results, 18 out of 32 i.e $56.25 \%$ had Caesarian deliveries, out of which 14 (77.78\%) had LSCS done for fetal distress.

Thus, the rate of LSCS and LSCS done for fetal distress in the present study correlates well with that of the Geetha et al study. ${ }^{13}$

The study shows that, incidence of LSCS and incidence of fetal distress as indication of LSCS is more in abnormal traces.

The study conducted by Vinita das et $\mathrm{al}^{12}$ and showed that, out of 175 cases studied, 129 had Reactive Admission test pattern, in which $8(6.2 \%)$ had meconium stained liquor, 2 (1.6\%) had Apgar score $<7$ @ 5 mins , 5 (3.9\%) had NICU Admissions and there were $2(1.6 \%)$ perinatal mortality. Out of 175 cases, 46 had Abnormal Admission test results, including both Non reassuring and Ominous AT patterns; in which 13
(28.26\%) had meconium stained liquor,(only moderate and thick meconium was considered); 4 (8.7\%) had

Apgar score < 7 @ mins, 7 (15.2\%) had NICU Admissions and there were $2(4.3 \%)$ cases of perinatal mortality.

Whereas in present study, out of 200 cases, 168 had Reassuring Admission test results, in which 8 (4.7\%) had meconium stained liquor, 2 (1.1\%) had Apgar score $<7 @ 5$ mins, 6 (3.5\%) had NICU Admissions and there was no perinatal mortality observed in our study. Out of 200 cases, 32 had Abnormal Admission test results, including both non reassuring and ominous AT patterns; in which 14 (43.25\%) had meconium stained liquor, 4 (12.5\%) had Apgar score $<7$ @ mins, 5 (15.6\%) had NICU Admissions and there was no perinatal mortality observed in our study.

The comparison shows that the individual fetal outcome parameters of the present study correlates well with those of Vinita das et al study. ${ }^{12}$

The study conducted by Aparna hegde et al ${ }^{1}$ with 200 low risk cases shows a sensitivity of $66.7 \%$, specificity of $90 \%$, PPV $38.7 \%$ and NPV of $96 \%$.

The study conducted by Kushtagi et al ${ }^{10}$ with 500 high and low risk cases shows a sensitivity of 53\%, specificity of $93 \%$, PPV $61 \%$ and NPV $91 \%$.

The study conducted by Ingemarsson et $\mathrm{al}^{3}$ included only low risk pregnancies, showed a sensitivity of $23.5 \%$, specificity of $99.4 \%$, PPV $40 \%$ and NPV $98.7 \%$.

The study conducted by Vinita das et al ${ }^{12}$ with 175 high and low risk cases shows a sensitivity of $38 \%$, specificity of $79 \%$, PPV $48 \%$ and NPV $72 \%$.

The present study has sensitivity of $60 \%$, specificity of $90.86 \%$, PPV $48.39 \%$ and NPV of $94 \%$.

The above data shows that the results obtained in the present study are comparable with the results of Aparna hedge et al study1 and Kustagi et al study. ${ }^{10}$

The present study shows, admission test has high specificity and low sensitivity in predicting outcomes in high risk cases which is consistent with the study of Rahman et al (specificity 95\%) who have also assessed in high risk cases. ${ }^{14}$

\section{Conclusion}

Admission test is a good screening test for detection of intrapartum fetal distress. It has a Sensitivity of $60 \%$, Specificity of $90.86 \%$, PPV of 
$48.39 \%$, NPV of $94 \%$, Accuracy of $87 \%$ and $\mathrm{P}$ value $<0.001$ which is statistically significant.

Reassuring Admission test is an excellent predictor of healthy fetus. The incidence of fetal distress and operative delivery is significantly more in patients with non reassuring and ominous AT patterns in both high and low risk group.

There is not much difference in fetal outcomes in low risk group in comparison with high risk group. In low risk group also, the intrapartum fetal morbidity, fetal heart changes, fetal acidosis can occur significantly. So Admission test can be used as a screening test in all pregnant women in labour irrespective of the risk status. Admission test in effective in predicting fetal outcome parameters like NICU admissions, MSL, Apgar score <7 @ 5 mins.

Admission test is a simple, reliable, noninvasive screening test to detect fetal compromise present at the time of admission in all pregnant women in labour irrespective of low risk or high risk status. By doing Admission test, we can catagorise which patients need continous EFM and who can be managed by intermittent auscultation alone. This reduces the load of continous EFM. Admission test can detect fetal distress already present at admission and prevent unnecessary delay in intervention.

\section{Reference}

1. Hegde A, Kore S, Shrikrishna S, Ambiye VR, Vaidya PR. Admission test - screening test for the prediction of fetal outcome in labour. J Obstet Gynecol India.2001;51:40-3.

2. Das V, Katiyar N, Malik GK. Role of Admission test. J Obstet Gynecol India 2001;51:48-50.

3. Ingemarsson I, Arulkumaran S, Ingemarsson E, Tambyaraja RI, Ratnam SS. Admission test:A screening test for fetal distress in labour. Obstet Gynecol 1986;68:800-906.

4. Royal college of obstetricians and gynaecologists. The use of electronic foetal monitoring. London: RCOG Press;2001.

5. Nordstrom L, Stockholm WU. Socialstyrelsen. Handläggning av normal födsel (Management of normal labour). 2001.

6. Gurung G, Rana A, Giri K. Detection of intrapartum fetal hypoxia using admission test (AT). Nepal Journal of Obstetrics and Gynaecology. 2009;1:10-3.

7. Mires G, Williams F, Howie P. Randomised controlled trial of cardiotocography versus Doppler auscultation of fetal heart at admission in labour in low risk obstetric population. BMJ. 2001;322:1457-60.

8. Sandhu GS, Raju R, Bhattacharyya TK. Admission Cardiotocography Screening of High Risk Obstetric Patients. Med J Armed Forces India. 2008;64:43-5.

9. Elimian A, Lawlor P, Figueroa R, Wiencek V, Garry D, Quirk JG. Intrapartum assessment

10. of fetal well-being: Any role for a fetal admission test? J Matern Fetal Neonatal Med. 2003;13:408-13.

11. Kushtagi $P$, Naragoni S. Labour admission test- an effective risk screening tool. J Indian Med Assoc 2002;100:234-6.

12. Buckshee K, Deka D, Padmaja V, Bhatla N. Admission test as a predictor of fetal outcome. J Obstet Gynecol India 1999;49:36-7.
13. Das V, Katiyar N, Malik GK. Role of Admission test. J Obstet Gynecol India 2001;51:48-50.

14. Geetha gurung, Ashma rana, Kanthi giri. Detection of intrapartum fetal hypoxia using Admission test. N. J. Obstet Gynecol 2006;17(2):10-3.

15. Hafizur R, Renjhen P, Dutta S, Kar S. Admission cardiotocography: its role in predicting foetal outcome in high-risk obstetric patients. Am Med J. 2012;5(10):522-7. 